\title{
Quantification of induced resistance against Phytophthora species expressing GFP as a vital marker: $\beta$-aminobutyric acid but not BTH protects potato and Arabidopsis from infection
}

\author{
AZEDDINE SI-AMMOUR ${ }^{a}$, BRIGITTE MAUCH-MANIb AND FELIX MAUCH* \\ University of Fribourg, Department of Biology, Pérolles, 1700 Fribourg, Switzerland
}

Published in "Molecular Plant Pathology 4(4): 237-248,2003"

which should be cited to reference this work.

\section{SUMMARY}

Induced resistance was studied in the model pathosystem Arabidopsis-Phytophthora brassicae (formerly P. porri) in comparison with the agronomically important late blight disease of potato caused by Phytophthora infestans. For the quantification of disease progress, both Phytophthora species were transformed with the vector p34GFN carrying the selectable marker gene neomycine phosphotransferase (nptll) and the reporter gene green fluorescent protein ( $g f p$ ). Eighty five per cent of the transformants of $P$. brassicae and $P$. infestans constitutively expressed GFP at high levels at all developmental stages both in vitro and in planta. Transformants with high GFP expression and normal in vitro growth and virulence were selected to quantify pathogen growth by measuring the in planta emitted GFP fluorescence. This nondestructive monitoring of the infection process was applied to analyse the efficacy of two chemical inducers of disease resistance, a functional SA-analogue, benzothiadiazole (BTH), and $\beta$ aminobutyric acid (BABA) which is involved in priming mechanisms of unknown nature. BABA pre-treatment $(300 \mu \mathrm{m})$ via soil drench applied $24 \mathrm{~h}$ before inoculation completely protected the susceptible Arabidopsis accession Landsberg erecta (Ler) from infection with $P$. brassicae. A similar treatment with BTH $(330 \mu \mathrm{m})$ did not induce resistance. Spraying the susceptible potato cultivar Bintje with BABA $(1 \mathrm{~mm}) 2$ days before inoculation resulted in a phenocopy of the incompatible interaction shown by the resistant potato cultivar Matilda while BTH (1.5 mM) did not protect Bintje from severe infection. Thus, in both pathosystems, the mechanisms of induced resistance appeared to be similar, suggesting that the Arabidopsis-P. brassicae pathosystem is a promising

\footnotetext{
*Correspondence: E-mail: felix.mauch@unifr.ch

Present addresses: ${ }^{a}$ Friedrich Miescher Institute, Maulbeerstrasse 66, CH-4058, Basel, Switzerland. ' UUniversity of Neuchâtel, Institute of Botany, Biochemistry Unit, Rue EmileArgand 9, PO Box 2, CH-2007 Neuchâtel, Switzerland.
}

model for the molecular analysis of induced resistance mechanisms of potato against the late blight disease.

\section{INTRODUCTION}

Phytophthora species cause very destructive diseases in a large number of plant species resulting in losses of several billions of dollars per year (Duncan, 1999; Erwin and Ribeiro, 1996; Kamoun, 2000). Diseases caused by Phytophthora species also cause great ecological damage. For example, $P$. cinnamomi destroyed large parts of the Jarrah Forest in Australia and P. ramorum, responsible for the sudden oak death epidemic in California, has recently spread to coastal redwoods (Knight, 2002). P. infestans, the agent responsible for the Irish potato late blight epidemics of the mid-19th century, still considerably constrains world potato and tomato production (Fry and Goodwin, 1997; Garelik, 2002; Judelson, 1997; Schiermeier, 2001). The situation is likely to worsen because of the appearance of highly aggressive strains of $P$. infestans resistant to the most widely used phenylamide fungicides and the recent spread of $A 2$ mating type isolates that will give rise to widespread sexual recombination (Duncan, 1999; Gisi and Cohen, 1996; Judelson, 1997; Shattock, 2002; Smart and Fry, 2001). The excessive use of pesticides to control plant diseases is a problem for today's plant production systems, and research priorities call for novel protection methods which are compatible with sustainable agriculture, thus favouring the use of alternative methods such as the application of chemical inducers of resistance (Kuc, 2001). Chemical inducers of resistance usually have no direct antimicrobial activity and can be divided into three groups: inorganic compounds, natural organic compounds and synthetic compounds (Oostendorp et al., 2001). The benzothiadiazoles (Kunz et al., 1997) with benzo(1,2,3)thiadiazole-7-carbothioic acid S-methyl ester (BTH) as their best known representative, activate resistance via salicylic acid (SA) mediated defence signalling pathways (Friedrich et al., 1996; Lawton et al., 1996; Oostendorp et al., 2001). 
Many studies have indicated that $\mathrm{SA}$ is an important signalling factor in plant disease resistance, and systemic increases in SA levels are linked to the induction of systemic aquired resistance (SAR) against many pathogens (Delaney et al., 1994; Dempsey et al., 1999; Gaffney et al., 1993; Ryals et al., 1996). Defence gene expression and resistance of plants against infection by Phytophthora species was shown to be induced by treatment of plants with cell wall preparations of $P$. infestans (Doke et al., 1987), with elicitin (Keller et al., 1996a), jasmonic acid (Cohen et al., 1993), unsaturated fatty acids (Bostock et al., 1982; Cohen et al., 1991; Coquoz et al., 1995), BABA (Cohen et al., 1994), tobacco necrosis virus (Anfoka and Buchenauer, 1997) and mycorrhizal symbiosis (Cordier et al., 1998). However, the mechanisms of biologically or chemically induced resistance against Phytophthora have rarely been analysed and the importance of SA and SAregulated gene expression for the establishment of resistance remains controversial (Abad et al., 1997; Alexander et al., 1993; Keller et al., 1996b; Vleeshouwers et al., 2000; Yu et al., 1997).

The quantification of induced disease resistance is often performed with methods such as estimation of diseased area or evaluation of sporulation. Depending on the pathosystem, these measurements can be rather subjective and time-consuming. The availability of visual molecular markers has led to a new approach in the quantification of disease resistance. The transformation of Cladosporium fulvum with the uidA gene permitted then following of fungal infection at the microscopic level as well as the photometric quantification of GUS activity as a measure of fungal biomass (Wubben et al., 1994). The transformation of Phytophthora species with GUS has been reported (Bailey et al., 1993; Judelson et al., 1992, 1993; van West et al., 1998) but was rarely used for the monitoring of disease progression (Kamoun et al., 1998) or the analysis of promoter activity (van West et al., 1998). More recent approaches took advantage of the availability of the green fluorescent protein (GFP) as a vital marker. Several plant pathogenic fungi were stably transformed with this reporter gene (Dumas et al., 1999; Vanden Wymelenberg et al., 1997). With respect to oomycetes, only $P$. palmivora and $P$. parasitica var. nicotianae were transformed with GFP as a reporter gene (Bottin et al., 1999; van West et al., 1999).

We transformed $P$. brassicae and $P$. infestans with a newly constructed transformation vector containing, as a reporter, a codon-optimized gfp gene. The GFP-expressing Phytophthora transformants were characterized and used to develop methods for the quantification of Phytophthora biomass in infected plants. The new disease quantification method was used to analyse chemically induced resistance of Arabidopsis against $P$. brassicae in comparison with the agronomically important late blight disease of potato caused by $P$. infestans. Our results show that BABA protected susceptible cultivars of both species from infection while BTH did neither protect Arabidopsis against $P$. brassicae nor potato against $P$. infestans. (a)

p34GFN
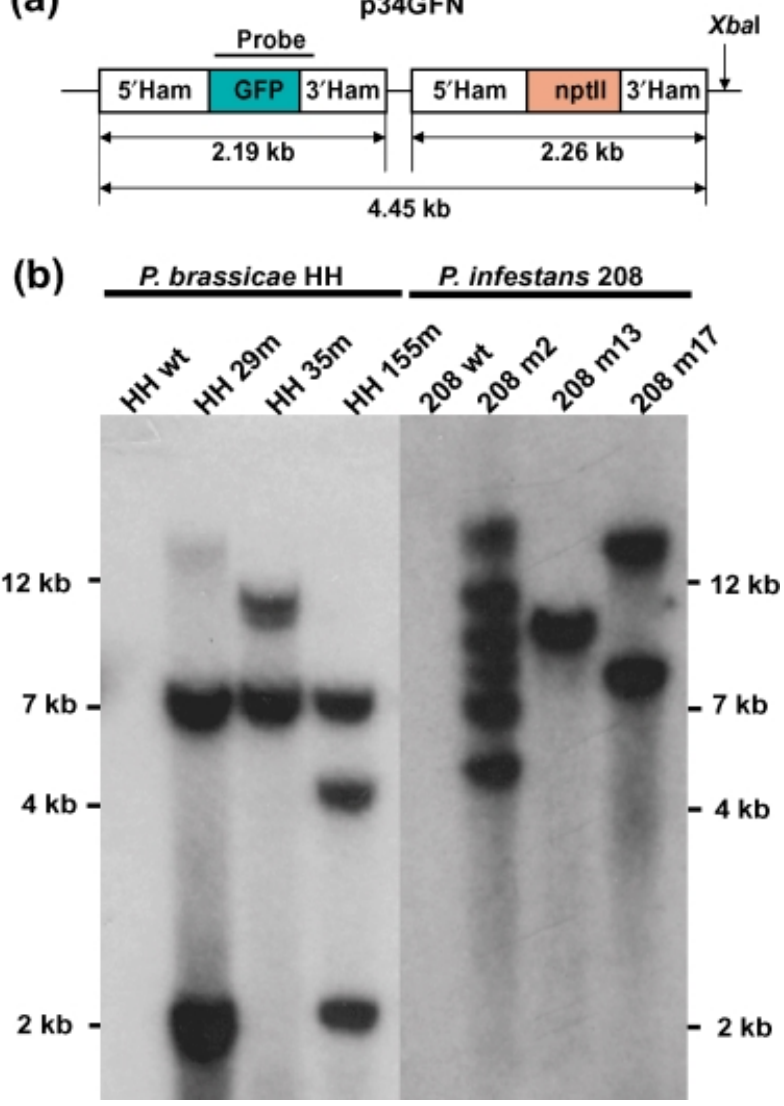

Fig. 1 Transformation construct p34GFN and Southern analysis of genomic DNA isolated from transformed Phytophthora. (a) Schematic view of the transformation vector $\mathrm{p} 34 \mathrm{GFN}$ containing a selectable marker cassette (npt/l) and a reporter gene cassette $(g f p)$. The $X b a l$ site and the probe used for Southern analysis are indicated. (b) Five $\mu \mathrm{g}$ of the genomic DNA of $P$. brassicae and $P$. infestans wild-type and of three p34GFN-transformants of each species were digested with $X b a l$, separated by electrophoresis and blotted on to nylon membranes. The blots were hybridized with a ${ }^{32} \mathrm{P}$-labelled probe specific for $g f p$. The $7.4 \mathrm{~kb}$ hybridizing band present in the restricted DNA of $P$. brassicae transformants corresponded to the size of p34GFN and indicates tandem integrations of the $\mathrm{p} 34 \mathrm{GFN}$ construct.

\section{RESULTS}

\section{Construction of the GFP-expression vector P34GFN and transformation of $\boldsymbol{P}$. brassicae and $\boldsymbol{P}$. infestans}

The transformation vector p34GFN (Fig. 1A) was used to transform both $P$. brassicae and $P$. infestans. This vector is a pBluescript II SK derived plasmid with two expression cassettes. One cassette contained the selectable marker gene neomycine phosphotransferase (nptII) and the other contained the reporter gene $g f p$. Both $n p t / l$ and $g f p$ were fused to promoter and terminator sequences of the ham34 gene of Bremia lactucae (Judelson et al., 1991). Among several GFP versions tested, a synthetic GFP with 
a S65T mutation in the chromophoric SYG tripeptide sequence and an increased C/G-content (Pang et al., 1996) was found to be most suitable as a visible marker in transgenic Phytophthora.

In preliminary transformation assays, co-transformation of $P$. brassicae using equal amounts (10 $\mu \mathrm{g}$ of each vector DNA) of the vector pHAM34N (Judelson et al., 1991) and the vector p34GF (see Experimental procedures) yielded only a few G418 resistant transformants and only $20 \%$ of them showed fluorescence which irreversibly disappeared during subcultivation. Compared to cotransformation with pHAM34N and p34GF, the transformation rate of $P$. brassicae was threefold higher using the doublecassette construct p34GFN, and a strong stable fluorescence was observed in $85 \%$ of the transformants. Transformation was first established with $P$. brassicae, and the successful protocol was then applied to transform $P$. infestans. The only difference between the transformation protocols for the two Phytophthora spp. was the starting material. Sporangia were used for $P$. infestans and, because the sporangia did not easily detach from the sporangiophores, zoospores were used for $P$. brassicae. A minimum of $1 \times 10^{4}$ zoospores for $P$. brassicae and $2 \times 10^{5}$ sporangia for $P$. infestans germinating in $10 \%(\mathrm{v} / \mathrm{v})$ V8 or ALBA medium, respectively, were required to obtain sufficient young mycelium (1 g) for protoplast production. The ratio of liposomes to protoplasts was found to be critical for obtaining high transformation efficiencies. Using the optimized protocol described in Experimental procedures, $P$. brassicae was transformed with an efficiency of 1-2.25 transformants per $\mu \mathrm{g}$ DNA and the transformation efficiency for $P$. infestans was on average 0.5 transformant $/ \mu \mathrm{g}$ DNA (Table 1). In total, 360 transformants of $P$. brassicae and 25 transformants of $P$. infestans were produced.

\section{Analysis of GFP-expressing Phytophthora transformants}

A Southern blot analysis was performed to demonstrate stable integration of p34GFN into the genome and to analyse the integration events (Fig. 1B). DNA isolated from wild-type and transformed $P$. brassicae and $P$. infestans, respectively, was digested with $X b$ al which cuts once within the p34GFN sequence at the $3^{\prime}$ end of the nptll cassette (Fig. 1A). Hybridization of the restricted DNA of $P$. brassicae transformants to a radioactively labelled probe specific for the GFP coding sequence revealed a strongly hybridizing band of the size of the linearized vector $(7.4 \mathrm{~kb})$ in many transformants, indicating a tandem integration of the p34GFN construct. Smaller and larger hybridizing fragments suggested the presence of one or two additional unlinked copies of the vector in the genome. Tandem integrations were less frequent in transformed $P$. infestans, and the transformants contained from 1 to a maximum of 6 independent copies of the vector sequence (Fig. 1B).

Eighty five per cent of the $P$. brassicae and $P$. infestans transformants showed a stable GFP fluorescence during their whole life cycle and GFP expression in both Phytophthora species was stable over time. Subculturing of the three transformants twice monthly over 2 years had no influence on the intensity of the GFP fluorescence. However, tests of in vitro growth revealed that the transformants often showed a reduced fitness compared to untransformed controls. Therefore, two $P$. brassicae isolate $\mathrm{HH}$ transformants ( $\mathrm{HH} 35 \mathrm{~m}$ and $\mathrm{HH} 155 \mathrm{~m})$ and one $P$. infestans CRA 208 transformant $(208 \mathrm{~m} 2)$ which showed normal in vitro growth and bright GFP fluorescence were selected for detailed characterization.

A comparison of the mycelial growth and production of sporangia, zoospores and cysts of $P$. brassicae transformants $\mathrm{HH} 35 \mathrm{~m}$ and $\mathrm{HH} 155 \mathrm{~m}$ and of $P$. infestans transformant $208 \mathrm{~m} 2$ revealed no difference in the respective wild-type strains. Temperature variations (from $12{ }^{\circ} \mathrm{C}$ to $30^{\circ} \mathrm{C}$ ) and elevated humidity (up to $100 \%$ ) had no effect on GFP expression. Figure 2 shows micrographs of the GFP expressing P. brassicae transformant HH35m at different developmental stages during in vitro growth and during infection of the susceptible Arabidopsis accession Ler. GFP fluorescence was high in in vitro grown zoospores (Fig. 2a), cysts (data not shown), mycelia (Fig. 2b) and sporangia (Fig. 2c,d). Similar
Table 1 Parameters and efficiency of Phytophthora transformation.

\begin{tabular}{lll}
\hline & ${\text { P. } \text { brassicae }^{\text {a }}}$ & P. infestans $^{\text {b }}$ \\
\hline Starting material $^{\mathrm{c}}$ & $1 \times 10^{4}$ zoospores & $2 \times 10^{5}$ sporangia $^{\mathrm{d}}$ \\
Number of protoplats $^{\mathrm{d}}$ & $1.5 \times 10^{6}-0.5 \times 10^{7}$ & $2.5 \times 10^{6}-1 \times 10^{7}$ \\
Percentage of germination $^{\mathrm{e}}$ & $30-80 \%$ & $35-40 \%$ \\
Transformants per assaye $^{\mathrm{e}}$ & $27-45$ & $3-20$ \\
Transformants per $\mu \mathrm{g}$ of vector DNA $^{\mathrm{e}}$ & $1-2.25$ & $0.15-1$ \\
\hline
\end{tabular}

asolate HH (CBS782.97).

bIsolate CRA 208.

The indicated numbers are the minimum number of zoospores or sporangia required to produce enough mycelium $(\sim 1 \mathrm{~g})$ for one assay.

dThe number of protoplasts should be in this range to ensure an optimum ratio between liposomes and protoplasts.

eMinimum-maximum range taken from 20 experiments for $P$. brassicae and three experiments for $P$. infestans. 

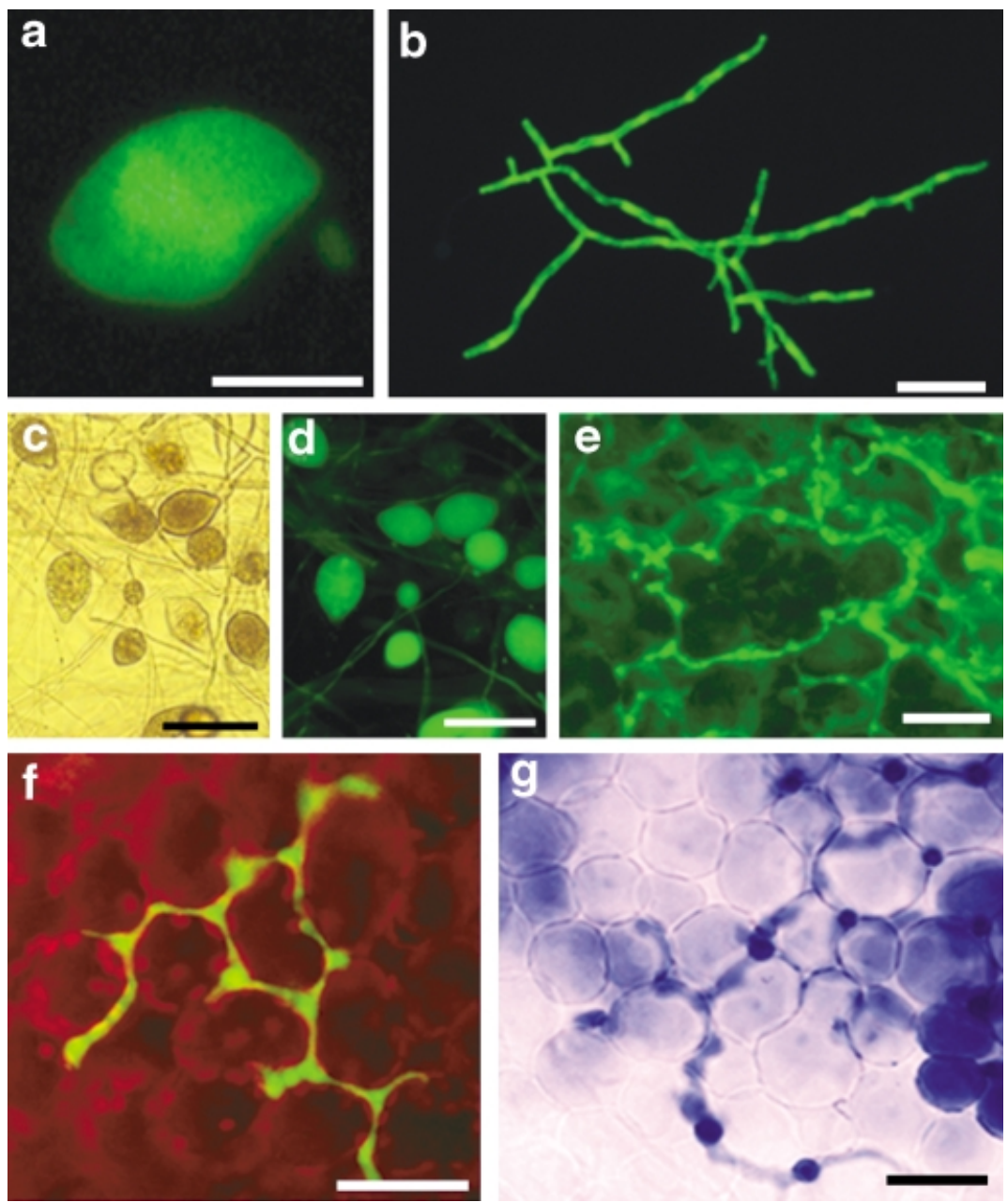

Fig. 2 Expression of GFP during different life stages of the $P$. brassicae transformant $\mathrm{HH} 35 \mathrm{~m}$. (a) Zoospore. Bar $=5 \mu \mathrm{m}$, (b) One-day-old germling growing on the surface of medium. Bar $=30 \mu \mathrm{m}$. (c) Bright field micrograph of zoosporangia on the surface of a mycelial mat. Bar $=50 \mu \mathrm{m}$. (d) Same as (c) but micrograph taken under blue light excitation. (e) Mycelium of $P$. brassicae inside a leaf of the susceptible $A$. thaliana accession Ler. The intercellularly growing hyphae denotes the shape of the plant cells. A barrier filter was used to absorb the red fluorescence from chlorophyll. Bar $=30 \mu \mathrm{m}$. (f) Mycelium of $P$. brassicae inside a leaf of $A$. thaliana accession Ler. Inside the plant cells, the red fluorescence from the chlorophyll is visible. Bar $=40 \mu \mathrm{m}$. (g) Infected leaf of $A$. thaliana accession Ler stained with lactophenol trypanblue. Dead plant cells and hyphae of $P$. brassicae are stained dark blue. Bar $=40 \mu \mathrm{m}$. results were found for $P$. infestans transformant 208m2 (Fig. 3). GFP was constitutively expressed in in vitro grown zoospores and cysts (data not shown), and germinating cysts (Fig. 3a), young mycelia (Fig. 3b), in sporangia (data not shown). High GFP fluorescence was also observed in all stages of pathogen development in susceptible Ler infected with P. brassicae transformant HH35m (Fig. 2e,f) and the susceptible potato cultivar Bintje infected with $P$. infestans 208m2 (Figs 3c,d, 5d,h). Confirming the initial biotrophic phase of both interactions, leaf tissue was initially colonized by mycelium growing in the intercellular space of Arabidopsis (Fig. 2e-g) and potato (Fig. 3c). Six days after infection, Arabidopsis and potato leaves were completely colonized and sporangiophores began to emerge from the stomates (as shown for potato in Fig. 3d). The major difference between the two interactions was that in infected potato plants the number of sporangiophores was much higher than in Arabidopsis. Comparative monitoring of the infection process over 6 days revealed that the in planta development of the $P$. brassicae transformants $\mathrm{HH} 35 \mathrm{~m}$ and $\mathrm{HH} 155 \mathrm{~m}$ and of the $P$. infestans transformant $208 \mathrm{~m} 2$ did not differ from the development of the wild-type strains.

\section{GFP-expressing Phytophthora as a tool for the analysis of induced resistance}

GFP-expressing $P$. brassicae transformant $\mathrm{HH} 35 \mathrm{~m}$ or $P$. infestans transformant $208 \mathrm{~m} 2$, respectively, were used to quantitatively test the potential of the two compounds BTH and BABA to induce disease resistance in susceptible Arabidopsis and potato plants. The effect of the treatments on resistance towards Phytophthora were analysed by the determination of in planta emitted GFP fluorescence in infected leaves (Fig. 4), and by measuring disease symptoms by fluorescence microscopy (Fig. 5). Figure 4a shows that the susceptible Arabidopsis accession Ler was not protected from infection by a pre-treatment with BTH (330 $\mu \mathrm{M}) 1$ day before inoculation. Six days after inoculation, the GFP-fluorescence emitted from BTH-treated leaves was comparable to that emitted from untreated inoculated leaves of Ler. In contrast, the fluorescence emitted from leaves of Ler pre-treated with BABA (300 $\mu \mathrm{m}) 1$ day before inoculation was only slightly higher than in uninoculated plants and similar to the fluorescence emitted by the resistant Arabidopsis accession Col. The 
Fig. 3 Expression of GFP during different life stages of the $P$. infestans transformant $208 \mathrm{~m} 2$. (a) Germinated cyst of $P$. infestans with a germ tube and an appressorium-like structure on the surface of the medium. The nucleus can be seen as a brighter spot inside the appressorium. Bar $=5 \mu \mathrm{m}$. (b) One-day-old germling on the surface of medium. Bar $=30 \mu \mathrm{m}$. (c) Fan-like growth of the mycelium in a leaf of potato cv. Bintje, $48 \mathrm{~h}$ post-inoculation. A barrier filter was used to absorb the red fluorescence from chlorophyll. Bar $=30 \mu \mathrm{m}$. (d) Sporangiophores and lose sporangia on the surface of a potato leaf cv. Bintje 6 days post-inoculation. The red fluorescence of chlorophyll is apparent in the background. Bar $=300 \mu \mathrm{m}$.
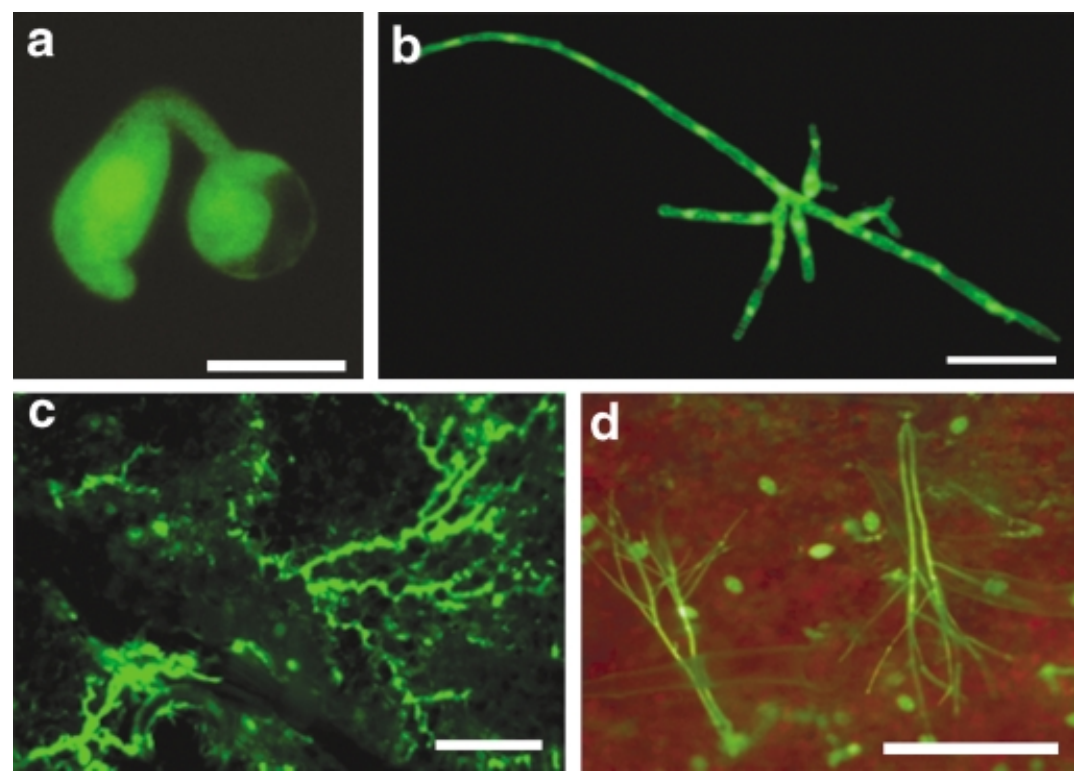

level of fluorescence emission coincided with the observed macroscopic symptoms and microscopic assessment of colonization (data not shown).

Measurement of in planta emitted fluorescence by $P$. infestans transformant $208 \mathrm{~m} 2$ showed that pre-treatment of the susceptible potato cultivar Bintje with BTH $(1.5 \mathrm{~mm})$ two days before inoculation did not effectively protect the plants from infection (Fig. 4b). Leaves of non-treated susceptible control plants showed typical symptoms of late blight infection with copious sporulation at the surface (Fig. 5c). Analysis by fluorescence microscopy revealed that the leaves were fully infected and the surface was covered with detached sporangia (Fig. 5d). Leaves of plants that had been pre-treated with BTH prior to inoculation with $P$. infestans displayed the same disease symptoms as non-induced plants, at the macroscopic (Fig. 5g) and at the microscopic level (Fig. 5h).

Pre-treatment of the susceptible potato cv. Bintje with the BABA (1 mm) 2 days before inoculation, led to full resistance. Fluorescence emission 6 days after inoculation was comparable to

Fig. 4 Quantification of induced resistance by measurement of in planta emitted fluorescence by GFP-expressing Phytophthora. (a) The resistant Arabidopsis accession Col and the susceptible accession Ler were treated via soil drench with water, BABA $(300 \mu \mathrm{m})$ or BTH $(330 \mu \mathrm{m}) 1$ day prior to inoculation with $P$. brassicae transformant $\mathrm{HH} 35 \mathrm{~m}$, and in planta emitted fluorescence was measured 6 days after inoculation. (b) Leaves of the resistant potato cultivar Matilda and the susceptible cultivar Bintje were sprayed with water, BABA (1 mm) or BTH (1.5 mm) 2 days before inoculation with $P$. infestans transformant $208 \mathrm{~m} 2$ and in planta emitted fluorescence was measured 6 days after inoculation. The results represent the mean value and standard deviations of three independent experiments for Arabidopsis and four independent experiments for potato. Fluorescence emission of 20 leaf discs was measured per experiment.
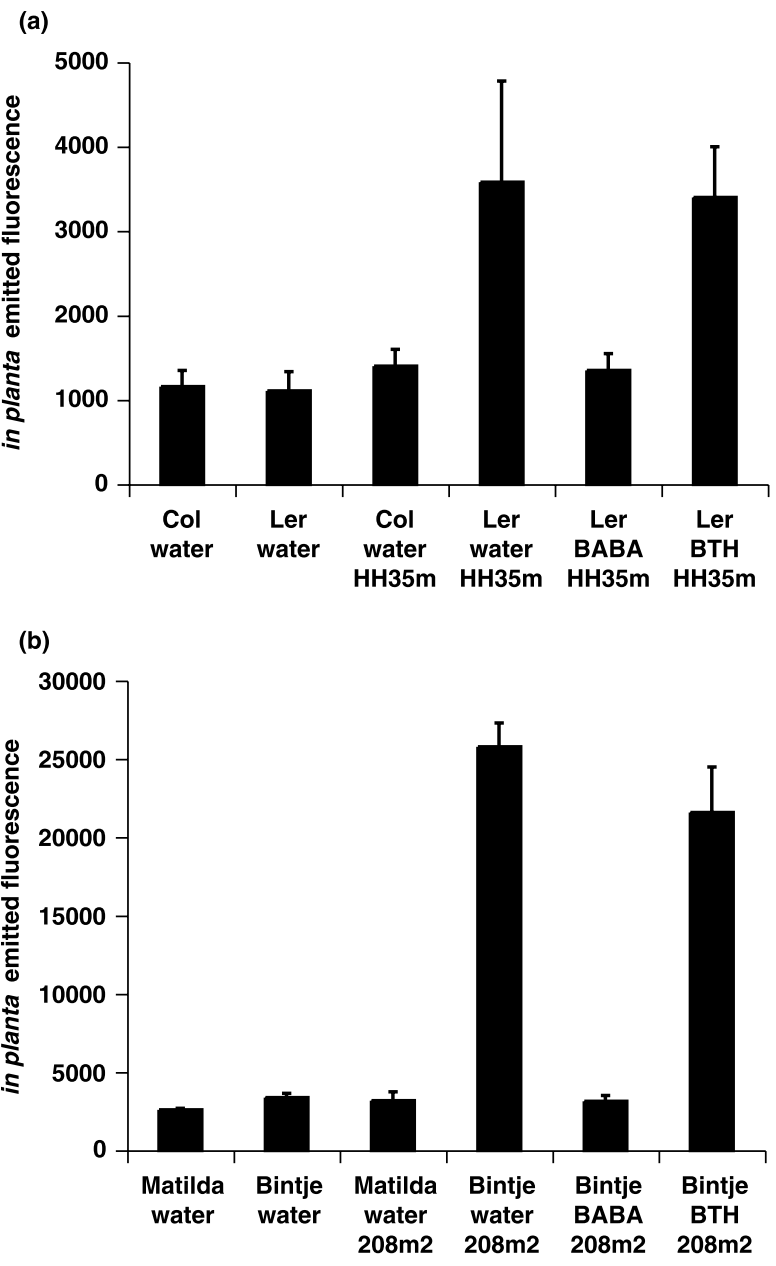

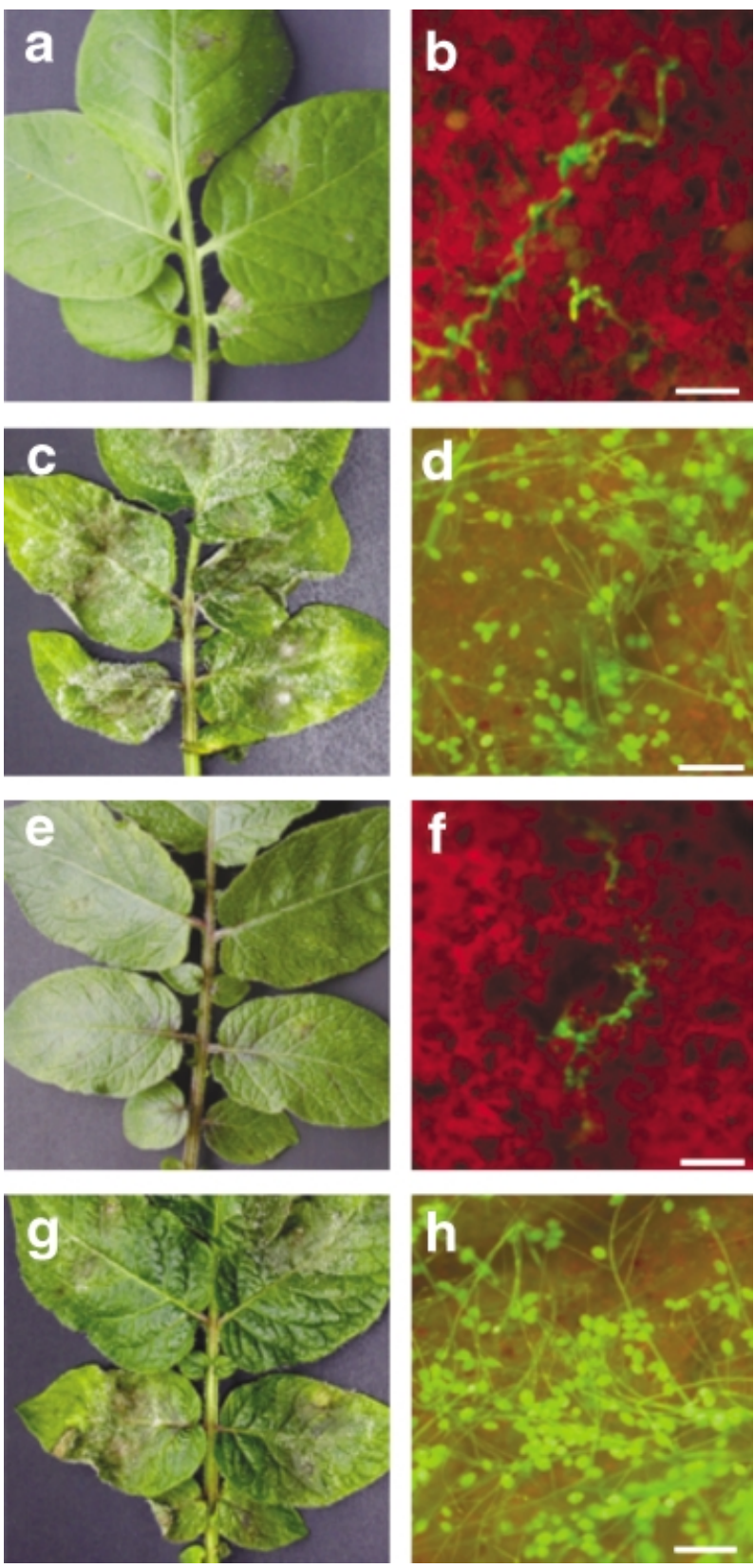

Fig. 5 Induction of resistance in potato towards $P$. infestans with BABA and BTH. All inoculations were made with $P$. infestans GFP-transformant 208m2, and photographs were taken 6 days after inoculation. (a) Leaf of the resistant potato cv. Matilda after inoculation with $P$. infestans. The small brown necrotic spots denote the inoculation sites. (b) Microscope view of the colonization of potato $\mathrm{cv}$. Matilda by $P$. infestans. Sparse bright green hyphae grow in the intercellular space. Bar $=100 \mu \mathrm{m}$. (c) Leaf of the susceptible potato cv. Bintje showing profuse white sporulation of $P$. infestans at the surface. (d) Microscope view of an infected area of a leaf of potato cv. Bintje infected with $P$. infestans. The leaf is covered with bright green sporiangiophores and sporangia. Reduced red chlorophyll fluorescence is a result of leaf necrosis. Bar $=100 \mu \mathrm{m}$. (e) Leaf of the susceptible potato $\mathrm{cv}$. Bintje pre-treated with $1 \mathrm{~mm}$ BABA 2 days before inoculation with $P$. infestans. A few necrotic spots denote the initial inoculation sites. (f) Microscope view of an area of a leaf of potato cv. Bintje pre-treated levels observed in the resistant potato cultivar Matilda (Fig. 4b). Inoculated leaves of Matilda displayed small necrotic areas at the site of attempted penetration by $P$. infestans (Fig. 5a). At the microscopic level, green fluorescing hyphae were rarely found and never developed to a stage of asexual reproduction (Fig. 5b). The same reaction type was observed in the susceptible potato plants Cv. Bintje pre-treated with BABA. Small necrotic flecks appeared at the site of attempted penetration (Fig. 5e), and almost no colonization by $P$. infestans was visible in the leaf tissue (Fig. $5 f$ ). Hence, pre-treatment of the susceptible potato cultivar Bintje with BABA led to a phenocopy of the resistant interaction with Matilda. BABA, at concentrations from 1 to $5 \mathrm{~mm}$, had no inhibitory effect on the in vitro growth of $P$. brassicae and $P$. infestans (data not shown).

\section{DISCUSSION}

We have previously described an Arabidopsis-P. brassicae pathosystem, and shown that genetic resistance in this system does not depend on functional SA, jasmonate or ethylene signalling (Roetschi et al., 2001). Here we describe an efficient transformation system for $P$. brassicae and the development of an optimized GFP reporter gene system that was used to visualize pathogen development in living plant tissue and to quantify pathogen biomass in infected leaves by measuring in planta emitted GFP fluorescence. A first application of the new tools to the analysis of induced resistance showed that the functional SA-analogue BTH (Kunz et al., 1997) did not protect Arabidopsis from infection by $P$. brassicae, thus suggesting that $S A$-mediated defence gene induction was insufficient to induce resistance against $P$. brassicae. This observation was extended to the late blight disease of potato caused by $P$. infestans. In contrast, Arabidopsis and potato plants were protected from infection by Phytophthora by pre-treatment with BABA, a compound that appears to be involved in priming mechanisms of unknown nature (Conrath et al., 2002; Zimmerli et al., 2000).

\section{Transformation of $\boldsymbol{P}$. brassicae and $\boldsymbol{P}$. infestans with a gfp reporter gene construct}

Several Phytophthora species were stably transformed with the reporter gene GUS (Bailey et al., 1993; Judelson et al., 1992;

with 1 mm BABA, 2 days before inoculation with $P$. infestans. Sporadic mycelial strands, recognized by their strong green fluorescence, can be seen growing in the intercellular space. Bar $=100 \mu \mathrm{m}(\mathrm{g})$ Leaf of the susceptible potato $\mathrm{cv}$. Bintje pre-treated with $1.5 \mathrm{~mm} \mathrm{BTH}, 2$ days before inoculation with $P$. infestans. Profuse sporulation and advanced necrosis are visible on the leaf. (h) Microscope view of an area of a leaf of potato cv. Bintje pre-treated with $1.5 \mathrm{~mm}$ BTH, 2 days before inoculation with $P$. infestans. The area is covered with bright green sporiangiophores and sporangia. Reduced red chlorophyll fluorescence is a result of leaf necrosis. Bar $=100 \mu \mathrm{m}$. 
Judelson, 1993; van West et al., 1998) but only one study reported the use of GUS expressing Phytophthora for the analysis of late blight resistance of potato (Kamoun et al., 1998). GFP was used as a reporter gene in transgenic $P$. palmivora and $P$. parasitica (Bottin et al., 1999; van West et al., 1999). However, the transformants were not used for disease quantification. We tested several versions of GFP to optimize the GFP reporter gene system for expression in Phytophthora. Possibly because Phytophthora ORFs are GC-rich (Qutob et al., 2000), synthetic GFPs (Pang et al., 1996) with an increased GC-content compared to the AT-rich wild-type sequence and a S65T mutation in the chromophoric SYG tripeptide sequence yielded the best results. GFP fluorescence of the $P$. brassicae and $P$. infestans transformants was readily visible by conventional fluorescence microscopy and was strong enough for detection in infected plants. The improved GFP reporter gene system has the potential to serve as a tool not only for disease quantification but more importantly for the analysis of gene regulation in Phytophthora.

Several procedures for the transformation of Phytophthora species have been described, including biolistic, electroporation, polyethylene-glycol/ $\mathrm{CaCl}_{2}$ - and liposome-mediated transformation (Bailey et al., 1993; Judelson et al., 1991, 1993). In our hands a protocol for the liposome-mediated transformation of Phytophthora (Judelson et al., 1991) with only minor modifications (see Experimental procedures) was the most reliable. As an improvement to the former co-transformation protocols (Bottin et al., 1999; Judelson et al., 1991, 1993; van West et al., 1998), a transformation vector (p34GFN) was constructed which contained both the selectable marker gene nptIl and $g f p$ each under the control of the ham 34 promoter of Bremia lactucae (Judelson et al., 1991, 1992). P. brassicae and P. infestans were successfully transformed with the new double cassette vector and a high percentage $(85 \%)$ of the geneticin resistant transformants showed GFP fluorescence. Although no detailed comparative analysis between the co-transformation method and transformation with p34GFN was performed, preliminary experiments showed that transformation with the double cassette vector was threefold more efficient than co-transformation and resulted in an increased stability of transgene expression. Similar to the results achieved with co-transformation (Bottin et al., 1999; Judelson et al., 1991, 1993; van West et al., 1999), frequent tandem integration of the transformation vector and multiple insertion sites were observed in our transformants.

For reasons that are not yet clear, the transformation efficiency achieved with $P$. brassicae was consistently higher than with $P$. infestans (Table 1). In the transformants that were analysed in more detail, GFP expression was stable for 2 years with frequent subculturing. However, many transformants showed decreased rates of in vitro growth. This frequent phenotype did not appear to be caused by the expression of the transgene, because no correlation between the intensity of GFP fluorescence and reduced growth was observed. We are currently testing whether the growth phenotype is a result of the procedures used in the transformation protocol and whether the affected transformants will recover over time.

\section{Induced resistance against Phytophthora}

The ham34 promoter driving gfp expression in p34FN was demonstrated to be constitutively active in Phytophthora species (Bottin et al., 1999; Judelson et al., 1991; van West et al., 1998, 1999). It conferred high GFP expression in the zoospores, cysts, hyphae and sporangiophores of $P$. brassicae (Fig. 2) and $P$. infestans (Fig. 3 ) both in vitro and in planta, thus providing a basis for the use of the visual marker for disease quantification. Transformants of $P$. brassicae and of $P$. infestans possessing high GFP expression, normal in vitro growth and unaltered virulence phenotype were used for the infection studies.

The functioning of SA in disease resistance against many pathogens is well established (Delaney et al., 1994; Gaffney et al., 1993; Ryals et al., 1996; Sticher et al., 1997). The role of SA in induced resistance against Phytophthora has been controversially discussed and may differ depending on the plant species. It is clear that the inoculation of various plant species including Arabidopsis and potato with Phytophthora triggers a rapid increase in SA levels, resulting in the activation of a number of putative defence genes such as PR1 (Coquoz et al., 1995; Roetschi et al., 2001). However, the contribution of SA-mediated defence gene expression to resistance against Phytophthora is unclear. Many potato cultivars have high endogenous SA levels (Coquoz et al., 1995; Yu et al., 1997) and cultivars with high field resistance against $P$. infestans showed a tendency to contain higher amounts of conjugated SA compared to susceptible cultivars (Coquoz et al., 1995). Similarly, the high constitutive expression of the $P R 1$ gene appeared to correlate with high nonspecific resistance in potato (Vleeshouwers et al., 2000). The purified PR1 protein of tomato and tobacco inhibited the germination of $P$. infestans zoospores in vitro and lesion growth in vivo (Niderman et al., 1995). In line with this observation, the over-expression of PR1 provided tolerance to tobacco plants against infection with P. parasitica (Alexander et al., 1993) and over-expression of PR5 slightly enhanced the resistance of potato against $P$. infestans (Liu et al., 1994). Engineered potato plants with lesion mimic phenotypes and high constitutive PR1 expression showed increased resistance against $P$. infestans (Abad et al., 1997; Tadege et al., 1998). Finally, tobacco plants expressing the bacterial salicylate hydroxylase gene (nahG) showed an enhanced susceptibility to several Phytophthora species (Keller et al., 1996b). In contrast, a drastic reduction in total SA levels in transgenic nah $G$ potato did not lead to increased disease susceptibility against $P$. infestans, suggesting that high basal levels of $S A$ are not causally linked to constitutive resistance (Yu et al., 1997). The application of exogenous SA did not increase the resistance of 
potato against $P$. infestans (Coquoz et al., 1995). In Arabidopsis, genetic resistance against $P$. brassicae (formerly $P$. porri; Man in't Veld et al., 2002) was independent of SA, jasmonate and ethylene and it was proposed that the resistance response is controlled by as-yet unknown signalling mechanisms (Roetschi et al., 2001). In support of this conclusion, the partial resistance of tomato against $P$. infestans was also found to be independent of signalling pathways controlled by the three stress hormones (Smart et al., 2003).

To test the contribution of SA-mediated defence signalling, we evaluated the effect of BTH on the disease resistance of potato and Arabidopsis against Phytophthora by quantifying pathogen biomass inside the leaf tissue using transgenic Phytophthora constitutively expressing GFP. BTH is well known as an activator of SA-mediated defence signalling pathways whose disease resistance inducing properties are dependent on a functional NPR1 gene (Lawton et al., 1996). Our results show that BTH is neither in potato an efficient inducer of resistance against $P$. infestans (Figs $4 \mathrm{~b}$ and 5 ) nor in Arabidopsis against $P$. brassicae (Fig. 4a). In both interactions, BTH pre-treatment did not prevent the colonization of the leaves and did not lead to reduced asexual sporulation. The concentration of BTH used in our experiments was $330 \mu \mathrm{m}$ for Arabidopsis and $1.5 \mathrm{~mm}$ for potato. A BTH concentration of $300 \mu \mathrm{m}$ effectively triggered defence gene expression and increased disease resistance against a broad spectrum of pathogens in many plant species, including Arabidopsis (Friedrich et al., 1996; Lawton et al., 1996; Oostendorp et al., 2001). There is however, little information on BTH-induced gene induction and resistance in potato. Concentrations of BTH of between 200 and $400 \mu \mathrm{m}$ induced resistance of potato against Erysiphe cichoracearum, Alternaria solani and Fusarium semitectum (Bokshi et al., 2003). Our own unpublished results showed that $1.5 \mathrm{~mm} \mathrm{BTH}$ induced the expression of the potato defence marker gene StPR-1 (van't Klooster et al., 1999). Contrary to our negative results, BTH provided protection of tobacco against $P$. parasitica (Friedrich et al., 1996) and of pepper against $P$. capsici (Matheron \& Porchas, 2002).

Pre-treatment with the resistance inducer BABA induced full protection of Arabidopsis against $P$. brassicae and of potato against $P$. infestans (Figs 4 and 5). Similar to BTH, BABA is known to induce resistance against a broad spectrum of pathogens in many plant species (Cohen, 2002; Jakab et al., 2001). In tomato, BABA sprayed on leaves provided a protection of $90 \%$ against $P$. infestans (Cohen et al., 1994) and partial protection of potato against $P$. infestans by BABA was reported in field experiments (Cohen, 2002). However, BABA was applied at high concentrations of up to $20 \mathrm{~mm}$ and it remained unclear whether the protective effect was an indirect result of BABA-induced necrosis. As noted before in tobacco and tomato (Cohen et al., 1994; Siegrist et al., 2000), high concentrations of BABA (10 mm) caused necrotic flecks on potato and tomato leaves, while no lesions were detected with a $1 \mathrm{~mm}$ solution of $B A B A$, which nonetheless conferred complete protection against $P$. infestans. In Arabidopsis, a soil drench treatment with $300 \mu \mathrm{m}$ BABA did not cause necrosis but was effective in disease protection. The lower limit of effective BABA concentration was not tested in our system. Concentrations of BABA as low as $80 \mu \mathrm{m}$ and $300 \mu \mathrm{m}$ induced resistance in Arabidopsis against Peronospora parasitica and Botrytis cinerea, respectively (Zimmerli et al., 2000, 2001). Upon pathogen inoculation, BABA-treated plants activated defence responses such as an increased production of PR1 and PDF1.2, much more rapidly than untreated controls (Zimmerli et al., $2000,2001)$. However, these two defence proteins were shown not to contribute to the resistance of Arabidopsis against $P$. brassicae (Roetschi et al., 2001). The general mechanism of BABA-mediated priming (Conrath et al., 2002) and the effector genes which confer BABA-induced resistance towards Phytophthora are not known.

The GFP expressing Phytophthora provide a tool for an accurate quantification of induced resistance. Inducers of disease resistance are rarely as effective or ineffective as the two compounds we have analysed here. Many biological and chemical inducers of disease resistance confer only different degrees of quantitative resistance, which is in most cases difficult to estimate objectively using previous methods. GFP-expresssing $P$. brassicae and $P$. infestans allow easier and more precise screening of potential inducers of disease resistance against Phytophthora in Arabidopsis, potato and possibly tomato.

\section{EXPERIMENTAL PROCEDURES}

\section{Transformation vector}

The GFP gene was excised as a Ncol/EcoRl fragment from pMON30060 (Pang et al., 1996), and inserted in the Smal site of plasmid pHAM34H (Judelson et al., 1991). The cassette containing GFP was then subcloned as a Hindlll/ECoRI fragment into pBluescript II SK (Stratagene, La Jolla, USA) yielding the p34GF plasmid. The cassette containing the $n p t / l$ gene fused to the ham34 promoter and terminator was cut from pHAM34N (Judelson et al., 1991) as a HindIII/EcoRI fragment and inserted in the pGEM-7Zf(+) plasmid (Promega, Madison, USA). The cassette was then cut out from the resulting plasmid and inserted as a $B a m H I / X b a l$ fragment into the P34GF plasmid giving the final transformation vector p34GFN (Fig. 1A) which contained the selectable $n p t / /$ marker and the visible marker gfp expressed under the control of the ham34 promoter and terminator sequences.

\section{Phytophthora culture conditions and transformation procedure}

$P$. brassicae isolate $\mathrm{HH}$ was grown and maintained as previously described (Roetschi et al., 2001). P. infestans isolate CRA 208 
was grown on rye sucrose agar medium (Caten and Jinks, 1967) at $18{ }^{\circ} \mathrm{C}$ in the dark. Both Phytophthora species were transformed according to the protocol of Judelson et al. (1991), with minor modifications. All steps were performed under aseptic conditions. Three-day-old mycelium was used to produce protoplasts. $P$. brassicae mycelium was initiated from a zoospore suspension in water obtained as previously described (Roetschi et al., 2001). The zoospores were induced to germinate by adding Campbell's V8 juice to a final concentration of $10 \%(\mathrm{v} / \mathrm{v})$. P. infestans mycelium was produced by supplementing a sporangial suspension in water with the same volume of $2 \times$ ALBA medium (Bruck et al., 1980). The later steps were the same for both Phytophthora spp. and the composition of the media used (KC, KC/MT and MT) is described in Judelson et al. (1991). Mycelium was washed in KC osmoticum and incubated for $35 \mathrm{~min}$ at room temperature in a solution of $\mathrm{KC}$ containing $5 \mathrm{mg} / \mathrm{mL}$ lysing enzymes from Trichoderma harzanium (Fluka, Buchs, Switzerland) and $2 \mathrm{mg} / \mathrm{mL}$ cellulase (Sigma Chemicals, St Louis, USA). The protoplasts were filtered through a $50 \mu \mathrm{m}$ nylon mesh, pelleted (700 g, $4 \mathrm{~min}$ ),

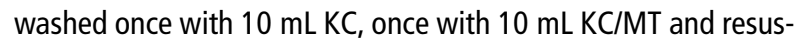
pended in MT medium to a final concentration of $1.5 \times 10^{6}-$ $0.5 \times 10^{7}$ protoplasts $/ \mathrm{mL}$ for $P$. brassicae and $2.5 \times 10^{6}-1 \times 10^{7}$ protoplasts $/ \mathrm{mL}$ for $P$. infestans. Sixty $\mu \mathrm{g}$ of lipofectin (Gibco BRL, Basel, Switzerland) was diluted in $400 \mu \mathrm{L}$ sterile water and kept for $30 \mathrm{~min}$ at room temperature and then mixed with $20 \mu \mathrm{g}$ vector DNA previously diluted in $250 \mu \mathrm{L}$ sterile water. After $15 \mathrm{~min}$ of incubation at room temperature, the $650 \mu \mathrm{L}$ of lipofectin-DNA mixture was added to $1 \mathrm{~mL}$ protoplast suspension. One $\mathrm{mL}$ of 50\% (w/v) PEG 3350 (Sigma Chemicals, St Louis, USA) was added, and after incubation for $5 \mathrm{~min}$, the mixture was diluted in $20 \mathrm{~mL}$ of $1 \mathrm{~m}$ mannitol containing $10 \%(\mathrm{v} / \mathrm{v}) \mathrm{V} 8$ medium for $P$. brassicae or $20 \mathrm{~mL}$ of $1 \mathrm{~m}$ mannitol containing rye sucrose medium, for $P$. infestans, respectively. The protoplasts germinated after $24 \mathrm{~h}$ incubation at $18{ }^{\circ} \mathrm{C}$ in the dark and were plated on to V8 agar containing $20 \mu \mathrm{g} / \mathrm{mL}$ geneticin (G418, Gibco BRL, Basel, Switzerland) for $P$. brassicae or rye sucrose agar containing $5 \mu \mathrm{g} / \mathrm{mL}$ geneticin for $P$. infestans. The regeneration rates were determined by microscope counting of serial dilutions of protoplasts plated on clarified V8 agar or clarified rye sucrose agar. The Phytophthora cultures (wild-type and transformants) were stored in liquid nitrogen as previously described (Roetschi et al., 2001). All transgenic Phytophthora and plants in contact with transgenic Phytophthora were autoclaved before disposal.

\section{Southern blot analysis}

Genomic DNA of Phytophthora was extracted with improved buffers for filamentous fungi genomic extraction using Qiagen genomic columns (Qiagen, Basel, Switzerland). Zoospores of $P$. brassicae were released (Roetschi et al., 2001) and resuspended in $10 \%(\mathrm{v} / \mathrm{v})$ V8 media for liquid culture. Sporangia of $P$. infestans were released in $10 \mathrm{~mL}$ of water and mixed with $10 \mathrm{~mL}$ of $2 \times$ ALBA medium (Bruck et al., 1980) for liquid culture. Mycelium was pulverized in liquid nitrogen, resuspended in lysis buffer (20 mm EDTA, $10 \mathrm{~mm}$ Tris-Cl (pH 7.9), 1 mg/mL lysing enzymes (Fluka, Buchs, Switzerland), 1\% (v/v) Triton X-100, 500 mm guanidine- $\mathrm{HCl}$ and $200 \mathrm{~mm} \mathrm{NaCl}$ ) and incubated at $37^{\circ} \mathrm{C}$ for $1 \mathrm{~h}$. Following treatment with DNAse-free RNAseA $(20 \mu \mathrm{g} / \mathrm{mL})$ and proteinase $\mathrm{K}(0.8 \mathrm{mg} / \mathrm{mL})$ the debris was pelleted by centrifugation and the clear lysate was transferred to a Qiagen genomic tip and processed as described by the manufacturer (Qiagen, Basel, Switzerland). Five $\mu \mathrm{g}$ of DNA was digested with $X b a l$, resolved on an agarose gel and transferred to a nylon membrane (Hybond- $\mathrm{N}$, Amersham Pharmacia Biotech, Little Chalfont, UK). The membrane was probed with ${ }^{32} \mathrm{P}$-radiolabelled gfp probe (RadPrime DNA Labeling System, Gibco BRL, Basel, Switzerland).

\section{Plant culture conditions and induction of resistance}

Arabidopsis accession Columbia (Col-0) and Landsberg erecta (Ler) were grown as previously described (Roetschi et al., 2001). Potato plants (Solanum tuberosum) cv. Bintje and cv. Matilda were grown from tubers (bought from Migros, Switzerland) in a growth chamber calibrated to $20{ }^{\circ} \mathrm{C}$ and $12 \mathrm{~h}$ of light $\left(100 \mu \mathrm{E} / \mathrm{m}^{2} /\right.$ s). Four-week-old Arabidopsis plants were treated by soil-drench with either $330 \mu \mathrm{m}$ BTH or $300 \mu \mathrm{m}$ BABA (Fluka, Buchs, Switzerland) as previously described (Zimmerli et al., 2001). One day after treatment, the plants were inoculated with plugs of V8 agar containing young growing mycelium, according to Roetschi et al. (2001). Potato compound leaves (4th to 6th) were cut from the plant and the petiole was inserted in wet stonewool cubes. Adaxial leaf surfaces were sprayed with either $1.5 \mathrm{~mm}$ BTH or BABA ( $1 \mathrm{~mm}$ or $10 \mathrm{~mm}$ ) with an atomizer. After 2 days, the treated leaves were transferred to a growth chamber maintained at $18^{\circ} \mathrm{C}$ with $10 \mathrm{~h}$ of light and infected with drops of $10 \mu \mathrm{L}$ water containing 250 sporangia. Complete darkness and high relative humidity were maintained for the first $24 \mathrm{~h}$ before the leaves were returned to $12 \mathrm{~h}$ of light and $20^{\circ} \mathrm{C}$.

\section{Measurements of in planta emitted GFP}

Six days after infection, leaf discs $(0.5 \mathrm{~cm}$ diameter) were punched out around the infection sites and placed, adaxial surface facing up, in a 96-mutliwell black maxisorp plate (Nunc, Wiesbaden, Germany) containing $200 \mu \mathrm{L}$ of sterile water in each well. The fluorescence emitted from the Phytophthora GFPtransformants growing in the leaf discs was measured with a fluorescence microplate reader Lambda Fluoro 320 (MWG Biotech, Ebersberg, Germany) using a static mode with 10 reads per well with an excitation wavelength of $485 / 20 \mathrm{~nm}$, an emission wavelength of 530/25 nm, and a sensitivity factor of 110 . The experiment was repeated threefold with Arabidopsis and $P$. brassicae 
and fourfold with potato and $P$. infestans. Twenty independent measurements were made for each treatment.

\section{Photographic documentation}

Phytophthora GFP-transformants were examined, in vitro or in planta, using a Leica DMR fluorescence microscope with different filter sets: $480 / 40 \mathrm{~nm}$ and $470 / 40 \mathrm{~nm}$ excitation GFP filters. Micrographs were taken using Kodak Ektachrome 400 film when a Leica MPS60 camera was used, or pictures were acquired using AxIovision 2.05 software and a Zeiss Axiocam CCD camera. Lactophenol trypan-blue staining was performed as previously described (Roetschi et al., 2001). Macroscopic symptoms were photographed with an Olympus Camedia digital camera.

\section{ACKNOWLEDGEMENTS}

The $P$. brassicae isolate $\mathrm{HH}$ was kindly provided by $\mathrm{F}$. Govers (University of Wageningen, the Netherlands) and the $P$. infestans isolate CRA 208 was kindly provided by D. Chavaillaz (Ciba-Geigy, Basel, Switzerland). BTH was a gift from U. Neuenschwander (Syngenta, Basel, Switzerland) and the synthetic GFP (pMON30060) was a gift from S. Pang (Monsanto, St Louis, USA). Special thanks go to $\mathrm{H}$. Judelson (University of California, Riverside, USA) for providing the initial transformation vectors and for his advice on the Phytophthora transformation. We thank G. Jakab for a critical reading of the manuscript. This project was financially supported by the Swiss National Science Foundation (grant no. 31-50519).

\section{REFERENCES}

Abad, M.S., Hakimi, S.M., Kaniewski, W.K., Rommens, C.M.T., Shulaev, V., Lam, E. and Shah, D.M. (1997) Characterization of acquired resistance in lesion-mimic transgenic potato expressing bacterioopsin. Mol. Plant-Microbe Interact. 10, 635-645.

Alexander, D., Goodman, R.M., Gut-Rella, M., Glascock, C., Weymann, K., Friedrich, L., Maddoy, D., Ahl-Goy, P., Luntz, T., Ward, E. and Ryals, J. (1993) Increased tolerance of two oomycete pathogens in transgenic tobacco expressing pathogenesis-related protein 1a. Proc. Natl Acad. Sci. USA, 90, 7327-7331.

Anfoka, G. and Buchenauer, H. (1997) Systemic acquired resistance in tomato against Phytophthora infestans by pre-inoculation with tobacco necrosis virus. Physiol. Mol. Plant Pathol. 50, 85-102.

Bailey, A.M., Mena, G.L. and Herrera-Estrella, L. (1993) Transformation of four pathogenic Phytophthora ssp by microprojectile bombardment on intact mycelia. Curr. Genet, 23, 42-46.

Bokshi, A.I., Morris, S.C. and Deverall, B.J. (2003) Effects of benzothiadiazole and acetylsalicylic acid on $\beta$-1,3-glucanase activity and disease resistance in potato. Plant Pathol. 52, 22-27.

Bostock, R.M., Lane, R.A. and Kuc, J.A. (1982) Factors affecting the elicitation of sesquiterpenoid phytoalexin accumulation by eicosapentaenoic and arachidonic acid in potato. Plant Physiol. 70, 1417-1424.
Bottin, A., Larche, L., Villalba, F., Gaulin, E., Esquerré-Tugayé, M.-T. and Rickauer, M. (1999) Green fluorescent protein (GFP) as gene expression reporter and vital marker for studying development and microbe-plant interaction in the tobacco pathogen Phytophthora parasitica var. nicotianae. FEMS Lett. 176, 51-56.

Bruck, R.I., Fry, W.E. and Apple, A.E. (1980) Effect of metalaxyl, an acylalanine fungicide on developmental stages of Phytophthora infestans. Phytopathology, 70, 597-601.

Caten, C.E. and Jinks, J.L. (1967) Spontaneous variability of single isolates of Phytophthora infestans. I. Cultural variation. Can J. Bot. 46, 329-348.

Cohen, Y. (2002) $\beta$-aminobutyric acid-induced resistance against plant pathogens. Plant Dis, 86, 448-457.

Cohen, Y., Gisi, U. and Mösinger, E. (1991) Systemic resistance of potato against Phytophthora infestans induced by unsaturated fatty acids. Physiol. Mol. Plant Pathol. 38, 255-263.

Cohen, Y., Gisi, U. and Niderman, T. (1993) Local and systemic protection against Phytophthora infestans induced in potato and tomato plants by jasmonic acid and jasmonic methyl ester. Phytopathology, 83, 10541062.

Cohen, Y., Niderman, T., Mösinger, E. and Fluhr, R. (1994) $\beta$-aminobutyric acid induces the accumulation of pathogenesis-related proteins in tomato (Lycopersicon esculentum L.) plants and resistance to late blight infection caused by Phytophthora infestans. Plant Physiol. 104, $59-66$.

Conrath, U., Pieterse, C.M.J. and Mauch-Mani, B. (2002) Priming in plant-pathogen interactions. Trends Plant Sci. 7, 210-216.

Coquoz, J.L., Buchala, A.J., Meuwly, P. and Métraux, J.P. (1995) Arachidonic acid treatment of potato plants induces local synthesis of salicylic acid and confers systemic resistance to Phytophthora infestans and Alternaria solani. Phytopathology, 85, 1219-1225.

Cordier, C., Pozo, O.J., Barea, J.M., Gianinazzi, S. and Gianinazzi-Pearson, V. (1998) Cell defense responses associated with localized and systemic resistance to Phytophthora parasitica induced in tomato by an arbuscular mycorrhizal fungus. Mol. Plant-Microbe Interact. 11, 1017-1028.

Delaney, T.P., Uknes, S., Vernooij, B., Friedrich, L., Weymann, K., Negrotto, D., Gaffney, T., Gut-Rella, M., Kessmann, H., Ward, E. and Ryals, J. (1994) A central role of salicylic acid in plant disease resistance. Science, 266, 1247-1250.

Dempsey, D.A., Shah, J. and Klessig, D.F. (1999) Salicylic acid and disease resistance in plants. Crit. Rev. Plant Sci. 18, 547-575.

Doke, N., Ramirez, A.V. and Tomiyama, K. (1987) Systemic induction of resistance in potato plants against Phytophthora infestans by local treatment with hyphal wall components of the fungus. J. Phytopathol. 119, 232-239.

Dumas, B., Centis, S., Sarrazin, N. and Esquérré-Tugayé, M.-T. (1999) Use of green fluorescent protein to detect expression of an endopolygacacturonase gene of Colletotrichum lindemuthianum during bean infection. Appl. Environ. Microbiol. 65, 1769-1771.

Duncan, J. (1999) Phytophthora-an abiding threat to our crops. Microbiol. Today, 26, 114-116.

Erwin, D.C. and Ribeiro, O.K. (1996) Phytophthora diseases worldwide. St Paul, MN: APS Press.

Friedrich, L., Lawton, K., Ruess, W., Masner, P., Specker, N., Gut-Rella, M., Meier, B., Dincher, S., Staub, T., Uknes, S., Metraux, J.-P., Kessmann, H. and Ryals, J. (1996) A benzothiadiazole derivative induces systemic acquired resistance in tobacco. Plant J. 10, 61-70.

Fry, W.E. and Goodwin, S.B. (1997) Re-emergence of potato and tomato blight in the United States. Plant Dis. 81, 1349-1357. 
Gaffney, T., Friedrich, L., Vernooij, B., Negrotto, D., Nye, G., Uknes, S., Ward, E., Kessmann, H. and Ryals, J. (1993) Requirement of salicylic acid for the induction of systemic acquired resistance. Science, 261, 754-756.

Garelik, G. (2002) Taking the bite out of potato blight. Science, 298, 17021704.

Gisi, U. and Cohen, Y. (1996) Resistance to phenylamide fungicides: a case study with Phytophthora infestans involving mating type and race structure. Annu. Rev. Phytopathol. 34, 549-572.

Jakab, G., Cottier, V., Toquin, V., Rigoli, G., Zimmerli, L., Métraux, J.-P. and Mauch-Mani, B. (2001) $\beta$-Aminobutyric acid-induced resistance in plants. Eur. J. Plant Pathol. 107, 29-37.

Judelson, H.S. (1993) Efficient cotransformation mediated by intermolecular ligation occuring in vivo in the oomycete, Phytophthora infestans. Mol. Gen. Genet. 239, 241-250.

Judelson, H.S. (1997) The genetics and biology of Phytophthora infestans: modern approaches to a historical challenge. Fungal Genet. Biol. 22, 65-76.

Judelson, H.S., Coffey, M.D., Arredono, F.R. and Tyler, B.M. (1993) Transformation of the oomycete Phytophthora megasperma f.sp. glycinea occurs by DNA integration into single or multiple chromosomes. Curr. Genet. 23, 211-218.

Judelson, H.S., Tyler, B.M. and Michelmore, R.W. (1991) Transformation of the oomycete pathogen, Phytophthora infestans. Mol. Plant-Microbe Interact. 4, 602-607.

Judelson, H.S., Tyler, B.M. and Michelmore, R.W. (1992) Regulatory sequences for expressing genes in oomycete fungi. Mol. Gen. Genet. 234, 138-146.

Kamoun, S. (2000) Phytophthora. In: Fungal Pathology (Kronstad, J.W., ed.), pp. 237-265. The Netherlands: Kluwer Academic.

Kamoun, S., van West, P. and Govers, F. (1998) Quantification of late blight resistance of potato using transgenic Phytophthora infestans expressing $\beta$-glucuronidase. Eur. J. Plant Pathol. 104, 521-525.

Keller, H., Blein, J.P., Bonnet, P. and Ricci, P. (1996a) Physiological and molecular characteristics of elicitin-induced systemic acquired resistance in tobacco. Plant Physiol. 110, 365-376.

Keller, H., Bonnet, P., Galiana, E., Pruvot, L., Friedrich, L., Ryals, J. and Ricci, P. (1996b) Salicylic acid mediates elicitin-induced systemic acquired resistance, but not necrosis in tobacco. Mol. Plant-Microbe Interact. 9, 696-703.

van't Klooster, J.W., Vleeshowers, V.G.A.A., Kamoun, S. and Govers, F. (1999) Characterization of a CDNA encoding a pathogenesis-related protein PR-1 from potato (Solanum tuberosum). Plant Physiol. 121, 1384.

Knight, J. (2002) Fears mount as oak blight infects red woods. Nature, 415, 251.

Kuc, J. (2001) Concepts and direction of induced systemic resistance in plants and its application. Eur. J. Plant Pathol. 107, 7-12.

Kunz, W., Schurter, R. and Maetzke, T. (1997) The chemistry of benzothiadiazole plant activators. Pesticide Sci. 50, 275-282.

Lawton, K.A., Friedrich, L., Hunt, M., Weymann, K., Delaney, T., Kessmann, H., Staub, T. and Ryals, J. (1996) Benzothiadiazole induces disease resistance in Arabidopsis by activation of the systemic acquired resistance signal transduction pathway. Plant J. 10, 71-82.

Liu, D., Ragtothama, K.G., Hasegawa, P.M. and Bressan, R.A. (1994) Osmotin overexpression in potato delays development of disease symptoms. Proc. Natl Acad. Sci. USA, 91, 1888-1892.

Man in't Veld, W.A., de Cock, A.W.A.M., llieva, E. and Lévesque, C.A. (2002) Gene flow analysis of Phytophthora porri reveals a new species: Phytophthora brassicae sp. nov. Eur. J. Plant Pathol. 108, 51-62.
Matheron, M.E. and Porchas, M. (2002) Suppression of Phytophthora root and crown rot on pepper plants treated with acibenzolar-S-methyl. Plant Dis. 86, 292-297.

Niderman, T., Genetet, I., Bruyère, T., Gees, R., Stintzi, A., Legrand, M., Fritig, B. and Mösinger, E. (1995) Pathogenesis-related PR-1 proteins are antifungal-Isolation and characterization of three 14-kilodalton proteins from tomato and of a basic PR-1 of tobacco with inhibitory activity against Phytophthora infestans. Plant Physiol. 108, 17-27.

Oostendorp, M., Kunz, W., Dietrich, B. and Staub, T. (2001) Induced disease resistance in plants by chemicals. Eur. J. Plant Pathol. 107, 19-28.

Pang, S.Z., DeBoer, D.L., Wan, Y., Ye, G., Layton, J.G., Neher, M.K., Armstrong, C.L., Fry, J.E., Hinchee, M.A.W. and Fromm, M.E. (1996) An improved green fluorescent protein gene as a vital marker in plants. Plant Physiol. 112, 893-900.

Qutob, D., Hraber, P.T., Sobral, B.W. and Gijzen, M. (2000) Comparative analysis of expressed sequences in Phytophthora sojae. Plant Physiol. $123,243-254$.

Roetschi, A., Si-Ammour, A., Belbahri, L., Mauch, F. and Mauch-Mani, B. (2001) Characterization of an Arabidopsis-Phytophthora pathosystem: resistance requires a functionnal $P A D 2$ gene and is independent of salicylic acid-, ethylene- and jasmonic acid-signaling. Plant J. 28, 293-305.

Ryals, J.A., Neuenschwander, U.H., Willits, M.G., Molina, A., Steiner, H.-Y. and Hunt, M.D. (1996) Systemic acquired resistance. Plant Cell, 8, 1809-1819.

Schiermeier, Q. (2001) Russia needs help to fend of potato famine, research warns. Nature, 410, 1001.

Shattock, R.C. (2002) Phytophthora infestans: populations, pathogenicity and phenylamides. Pest Manag. Sci. 58, 944-950.

Siegrist, J., Orober, M. and Buchenauer, H. (2000) $\beta$-aminobutyric acidmediated enhancement of resistance in tobacco to tobacco mosaic virus depends on the accumulation of salicylic acid. Physiol. Mol. Plant. Pathol. 56, 95-106.

Smart, C.D. and Fry, W.E. (2001) Invasion by the late blight pathogen. Renewed sex and enhanced fitness. Biol. Invasions, 3, 235-243.

Smart, C.D., Myers, K.L., Restrepo, S., Martin, G.B. and Fry, W.E. (2003) Partial resistance of tomato to Phytophthora infestans is not dependent upon ethylene, jasmonic acid, or salicylic acid signaling pathways. Mol. Plant-Microbe Interact. 16, 141-148.

Sticher, L., Mauch-Mani, B. and Métraux, J.-P. (1997) Systemic acquired resistance. Annu. Rev. Phytopathol. 35, 235-270.

Tadege, M., Bucher, M., Stähli, Suter, M., Dupuis, I. and Kuhlemeier, C. (1998) Activation of plant defense responses and sugar efflux by expression of pyruvate decarboxylase in potato leaves. Plant J. 16, 661-671.

Vanden Wymelenberg, A.J., Cullen, D., Spear, R.N., Schoenike, B. and Andrews, J.H. (1997) Expression of green fluorescent protein in Aureobasidium pullulans and quantification of the fungus on leaf surfaces. Biotechniques, 23, 686-690.

Vleeshouwers, V.G.A.A., van Dooijeweert, W., Govers, F., Kamoun, S. and Colon, L.T. (2000) Does basal PR gene expression in Solanum species contribute to non-specific resistance to Phytophthora infestans? Physiol. Mol. Plant Pathol. 57, 35-42.

van West, P., de Jong, A.J., Judelson, H.S., Emons, A.M.C. and Govers, F. (1998) The ipiO gene of Phytophthora infestans is highly expressed in invading hyphae during infection. Fungal Genet. Biol. 23, 126-138.

van West, P., Reid, B., Campbell, T.A., Sandrock, R.W., Fry, W.E., Kamoun, S. and Gow, N.A.R. (1999) Green fluorescent protein (GFP) as a reporter gene for the plant pathogenic oomycete Phytophthora palmivora. FEMS Lett. 178, 71-80. 
Wubben, J.P., Joosten, M.H. and De Wit, P.J. (1994) Expression and localization of two in planta induced extracellular proteins of the fungal tomato pathogen Cladosporium fulvum. Mol. Plant-Microbe Interact. 7, 516-524.

Yu, D., Liu, Y., Fan, B., Klessig, D.F. and Chen, Z. (1997) Is the high basal level of salicylic acid important for disease resistance in potato? Plant Physiol. 115, 343-349.
Zimmerli, L., Jakab, G., Métraux, J.-P. and Mauch-Mani, B. (2000) Potentiation of pathogen-specific defense mechanisms in Arabidopsis by $\beta$-aminobutyric acid. Proc. Natl Acad. Sci. USA, 97, 1292012925.

Zimmerli, L., Métraux, J.-P. and Mauch-Mani, B. (2001) $\beta$-aminobutyric acid-induced protection of Arabidopsis against the necrotrophic fungus Botrytis cinerea. Plant Physiol. 126, 517-523. 